\title{
El rol de la microbiota intestinal en la dermatitis atópica
}

\section{The role of the intestinal microbiota in atopic dermatitis}

\author{
Jessica Sulema Rodarte-Acosta, ${ }^{*}$ Martha Alejandra Morales-Sánchez ${ }^{\star}$
}

\section{RESUMEN}

La microbiota intestinal se define como la colección de microbios (bacterias, hongos, arqueas y virus) que habitan el intestino humano. El tracto gastrointestinal alberga aproximadamente $10^{14}$ microbios. La microbiota gastrointestinal es esencial para la maduración del sistema inmunológico, compuesto por respuestas inmunitarias tanto adaptativas como innatas. Los cambios en la composición y función microbiana se denominan disbiosis; se ha observado disbiosis en la patogenia de trastornos metabólicos, cáncer y enfermedades autoinmunes no intestinales y dermatológicas como dermatitis atópica, psoriasis y alopecia areata. La dermatitis atópica (DA) es una enfermedad crónica, autoinmune que afecta a $15 \%$ de la población infantil, existe una posible interacción entre los microbios y la dermatitis atópica de acuerdo a la hipótesis de la higiene. La disbiosis intestinal afecta la respuesta inmune cutánea de manera que los niños están predispuestos a una serie de alteraciones inmunológicas, capaz de desencadenar la activación inmunitaria y la liberación de citocinas implicadas en el desarrollo posterior de los signos clínicos de la DA. Aún está en debate si una barrera intestinal alterada facilita un mayor espectro de trastornos de hipersensibilización, como en la DA; sin embargo, la comprensión de la microbiota intestinal en las dermatosis inmunomediadas podría sugerir tratamientos adicionales además de las terapias convencionales.

Palabras clave: Dermatitis atópica, microbiota intestinal, atopia, dermatología, probióticos.

\section{ABSTRACT}

The intestinal microbiota is defined as the collection of microbes (bacteria, fungi, archaea, and viruses) that inhabit the human intestine. The gastrointestinal tract shelters approximately $10^{14}$ microbes. The gastrointestinal microbiota is essential for the maturation of the immune system, composed of both adaptive and innate immune responses. Changes in microbial composition and function are called dysbiosis; dysbiosis has been observed in the pathogenesis of metabolic disorders, cancer, and non-intestinal and dermatological autoimmune diseases such as atopic dermatitis, psoriasis, and alopecia areata. Atopic dermatitis $(A D)$ is a chronic, autoimmune disease that affects $15 \%$ of the children, there is a possible interaction between microbes and atopic dermatitis according to the hygiene hypothesis. Intestinal dysbiosis affects the cutaneous immune response in a way that children are predisposed to a number of immune conditions, capable of triggering immune activation and the release of cytokines involved in the subsequent development of clinical signs of $A D$. It is still debated whether an altered gut barrier facilitates a broader spectrum of hypersensitization disorders, such as $A D$, however, understanding the gut microbiota in immune-mediated dermatoses could suggest additional treatments in addition to conventional therapies.

Keywords: Atopic dermatitis, intestinal microbiota, atopy, dermatology, probiotics.

\section{INTRODUCCIÓN}

Los seres humanos han evolucionado en conjunto con los billones de microbios que habitan el cuerpo humano, creando ecosistemas adaptativos complejos y específicos, que están sincronizados con la fisiología del huésped en constante cambio. ${ }^{1}$

\footnotetext{
* Unidad de Educación e Investigación, Centro Dermatológico «Dr. Ladislao de la Pascua» (CDP), Ciudad de México, México.
}

La microbiota intestinal se define como la colección de microbios (bacterias, hongos, arqueas y virus) que habitan el intestino humano. ${ }^{2}$ Aproximadamente $10^{3}$ microorganismos habitan el tracto gastrointestinal, se estima que es más de 100 veces la cantidad de contenido genómico (microbioma) que el genoma humano. ${ }^{4}$

Citar como: Rodarte-Acosta JS, Morales-Sánchez MA. El rol de la microbiota intestinal en la dermatitis atópica. Rev Cent Dermatol Pascua. 2021; 30 (2): 76-83. https://dx.doi.org/10.35366/101177 
Los microbios del tracto gastrointestinal participan en una serie de procesos biológicos humanos, descomposición de alimentos, síntesis de vitaminas y biomoléculas, regulación de los sistemas inmunitarios innato y adaptativo. ${ }^{2,5,6}$

Los cambios en la composición y función microbiana son denominados disbiosis. La falta de diversidad en el microbioma intestinal se ha observado en enfermedades que van desde enfermedad inflamatoria intestinal, esclerosis múltiple, diabetes (tipos 1 y 2), alergias, asma, autismo y cáncer, ${ }^{7}$ hasta enfermedades dermatológicas como dermatitis atópica (DA), psoriasis y alopecia areata. ${ }^{1,8}$

La dermatitis atópica es una enfermedad sistémica inflamatoria crónica, autoinmune caracterizada por prurito intenso, que afecta a 15\% de la población pediátrica, $85-90 \%$ suele presentarse durante la infancia..$^{9,10}$ Aunque la patogenia del trastorno no se comprende completamente, se considera que existe desregulación inmunitaria. ${ }^{11,12}$

\section{MICROBIOTA INTESTINAL}

El microbioma se ha denominado "segundo genoma» debido al impacto que tiene en la salud humana,,$^{12}$ y muestra regularmente alto grado de diversidad interpersonal incluso en ausencia de enfermedad. ${ }^{1}$

Las funciones fisiológicas que ejerce la microbiota son: fortalecer la integridad intestinal o dar forma al epitelio intestinal, ${ }^{13}$ resistencia a la colonización por especies patógenas, ${ }^{14}$ síntesis de novo de vitaminas esenciales, ${ }^{3}$ homeostasis metabólica y desarrollo de la mucosa intestinal y del sistema inmunológico intestinal y sistémico. ${ }^{15,16}$

La microbiota gastrointestinal es esencial para la maduración del sistema inmunológico, compuesto por respuestas inmunitarias tanto adaptativas como innatas. La inmunidad innata se basa en la barrera física del epitelio; mientras que la inmunidad adaptativa ocurre a través de la estimulación de linfocitos que expresan anticuerpos específicos de reacción cruzada. Se ha demostrado que el desarrollo temprano de la microbiota intestinal es importante para la respuesta inmune normal y para prevenir enfermedades autoinmunes. ${ }^{16,17}$ La microbiota estimula y a su vez es estimulada por la inmunidad innata, equilibrio que puede ser alterado por patógenos. ${ }^{5}$

Las bacterias filamentosas desempeñan un papel clave en las respuestas de las células intestinales, equilibrando las respuestas celulares $T_{H} 1$ y $T_{H} 2$. Una microbiota saludable se asocia con incremento de la interleucina-17 (IL-17), participa en el mantenimiento e integridad de la barrera mucosa, secreta IL-17, IL-17F e IL-22, e influye en las células $T_{\text {reg }}$, productoras de IL-10, capaces de reconocer antígenos derivados de comensales. ${ }^{18}$

\section{Colonización microbiana}

La colonización microbiana continúa desarrollándose y modulando la cantidad de especies; alrededor de los dos a tres años de edad, la composición, diversidad y capacidades funcionales de la microbiota infantil se asemejan a las de la microbiota adulta. ${ }^{19}$ Los primeros tres años de vida representan el periodo más crítico, los niños experimentan cambios significativos en el desarrollo que influyen en su estado de salud y en su sistema inmunológico. ${ }^{19,20}$

En el intestino infantil, la colonización es de vital importancia para un crecimiento saludable, ya que influye en la maduración intestinal, el desarrollo metabólico, inmunológico y cerebral en la vida temprana. ${ }^{16}$ Los factores perinatales como el modo de parto, edad gestacional (prematuridad), dieta, genética y la glucosilación de la mucina intestinal contribuyen a influir en la colonización microbiana, y posteriormente a lo largo de la vida, el uso de antibióticos, área geográfica, contaminación ambiental y la dieta. . $^{1,21-24}$

Previamente se reconocía que la colonización intestinal en el recién nacido ocurría después del nacimiento; en la actualidad, se ha demostrado que la influencia temprana de la microbiota puede ser evidente in utero, ${ }^{20,21}$ ya que la colonización microbiana durante el embarazo regula el número de células inmunitarias innatas específicas y su actividad en los neonatos. ${ }^{25}$

El microbioma intestinal humano adulto está compuesto por más de 1,000 especies bacterianas, la mayoría son anaerobios obligados, dominado en $90 \%$ por los phyla Firmicutes y Bacteroidetes, seguido en menor proporción por los phyla Actinobacteria, Proteobacterias, Verrucomicrobia y Fusobacteria, y además contiene eucariotas, como Candida, Malassezia y Saccharomyces. ${ }^{4,26,27}$

Los phyla Proteobacterias y Bacteroidetes son bacterias gramnegativas involucradas en la digestión de carbohidratos, desarrollo del microbioma intestinal, modulación del sistema inmunológico y la protección contra la colonización por patógenos. ${ }^{2,5}$ Las especies pertenecientes a los phyla Bacteriodetes y Firmicutes pueden fermentar carbohidratos no digeribles, la fibra, para producir ácidos grasos de cadena corta (AGCC), 
ácidos grasos ramificados, lactato y etanol los cuales son la principal fuente de energía de las células epiteliales intestinales. ${ }^{5}$

Los primeros colonizadores, anaerobios facultativos, crean un nuevo ambiente que promueve la colonización de anaerobios estrictos como Bacteroides, Clostridium y Bifidobacterium spp. La microbiota fecal neonatal se caracteriza por una baja diversidad y está típicamente dominada por los phyla Proteobacterias y Actinobacterias, y por Bifidobacteriaceae y Enterobacteriaceae y la microbiota se vuelve más diversa con la aparición y el dominio de Firmicutes y Bacteroidetes a medida que pasa el tiempo después del nacimiento. ${ }^{19,28,29}$

En la primera semana de vida del recién nacido, el desarrollo de la microbiota intestinal es influenciado principalmente por la nutrición. ${ }^{22}$ Los recién nacidos alimentados con leche materna tienen un microbioma intestinal más diverso que los alimentados con biberón; Bifidobacterium spp es el microorganismo predominante en el primer grupo, su número está duplicado en niños alimentados con leche materna. ${ }^{30}$ Escherichia coli ( $E$. coli) y Clostridium difficile (C. difficile) predominan en los recién nacidos alimentados con biberón. ${ }^{31}$ La lactancia materna se asocia a un mejor desarrollo de células $T$ efectoras $17\left(T_{H} 17\right)$, que son un modulador importante de inmunidad inducido por las bacterias comensales intestinales. 22,32

Si bien la microbiota fecal de $72 \%$ de los bebés nacidos por vía vaginal se asemeja a la microbiota fecal de sus madres, en los bebés nacidos por cesárea este porcentaje se reduce a sólo $41 \% .{ }^{20}$ Algunas especies de Bifidobacterium y Bacteroides son muy abundantes en los bebés nacidos por parto vaginal, lo que permite la regulación a la baja de las respuestas inflamatorias; predominan las especies de Streptococcus y de Staphylococcus; $C$. difficile se encuentra en bebés nacidos por cesárea. ${ }^{33}$

Las partículas (PM) de la contaminación del aire se han asociado como factor de riesgo de disbiosis por su capacidad para inducir el estrés oxidativo y la liberación de citoquinas proinflamatorias, lo que aumenta la permeabilidad intestinal. ${ }^{34}$

\section{DERMATITIS ATÓPICA Y LA DISBIOSIS INTESTINAL}

En la patogenia de la DA, influye la disfunción de la barrera intestinal y la desregulación inmunitaria. ${ }^{15,35}$ La interacción crucial con la microbiota en la DA se representa mejor a través de la hipótesis de la higiene, la hipótesis de Strachan (1989), las condiciones «des- infectadas» de la vida moderna reducen la exposición microbiana en edad temprana de la vida, esto resulta en un desarrollo inmune inadecuado, siendo una posible causa del incremento de las enfermedades atópicas. Esta hipótesis está respaldada por los hallazgos con respecto a disminución en la diversidad del microbioma intestinal en los bebés con DA. ${ }^{33,36}$

La disbiosis intestinal afecta la respuesta inmune cutánea de manera que los niños están predispuestos a una serie de alteraciones inmunológicas, esto parece ser un evento temprano y duradero en los niños propensos a la DA, capaz de desencadenar la activación inmunitaria y la liberación de citocinas implicadas en el desarrollo posterior de los signos clínicos de la DA. ${ }^{2,33}$

\section{Composición de la microbiota en la dermatitis atópica}

Los niños con DA tienen poca diversidad de microorganismos, además de bajas cantidades de Bifidobacterium y Bacteroides; en contraste tienen elevados niveles de Enterobacteriaceae. ${ }^{33,36}$ La cantidad de tipos de microorganismos también influye en el curso y la severidad de la patología, bajos niveles de Streptococcus y altas cantidades de Akkermansia en casos de DA transitoria, y bajos niveles de Clostridium, Akkermansia y altos de Streptococcus en niños con DA persistente. La abundancia de Streptococcus se correlaciona positivamente con la puntuación de SCORAD para DA $^{37-39}$ (Figura 1).

Se observa disminución de las bacterias productoras de AGCC (Bifidobacterium, Blautia, Coprococcus, Eubacterium y Propionibacterium). La reducción de Bifidobacterium, Faecalibacterium prausnitzii y Coprococcus eutactus se ha observado en la DA con síntomas severos. Bifidobacterium puede estimular la producción de citocinas en respuesta a $T_{H} 1$, llevando a inmunidad dominada por éste. ${ }^{39-42}$

F. prausnitzii es el principal productor de butirato en el colon, fuente de energía para colonocitos con efectos antiinflamatorios y estimulación de células $T_{\text {reg }}$, lleva a una respuesta inmune aberrante tipo $\mathrm{T}_{H} 2$, lo que favorece la respuesta a alérgenos en la piel. ${ }^{43}$

El estudio de cohorte KOALA demostró que la presencia de $E$. coli y $C$. difficile se asocian con elevado riesgo de DA y otras patologías atópicas, debido a que alteran la diferenciación de células $\mathrm{T}^{44} \mathrm{E}$. coli puede evocar respuesta inflamatoria intestinal gracias a los lipopolisacaridos, conduce a mayor sensibilización atópica en la DA. ${ }^{45}$ Los AGCC (e.g. butirato, propionato y acetato) tienen efectos inmunomoduladores y 


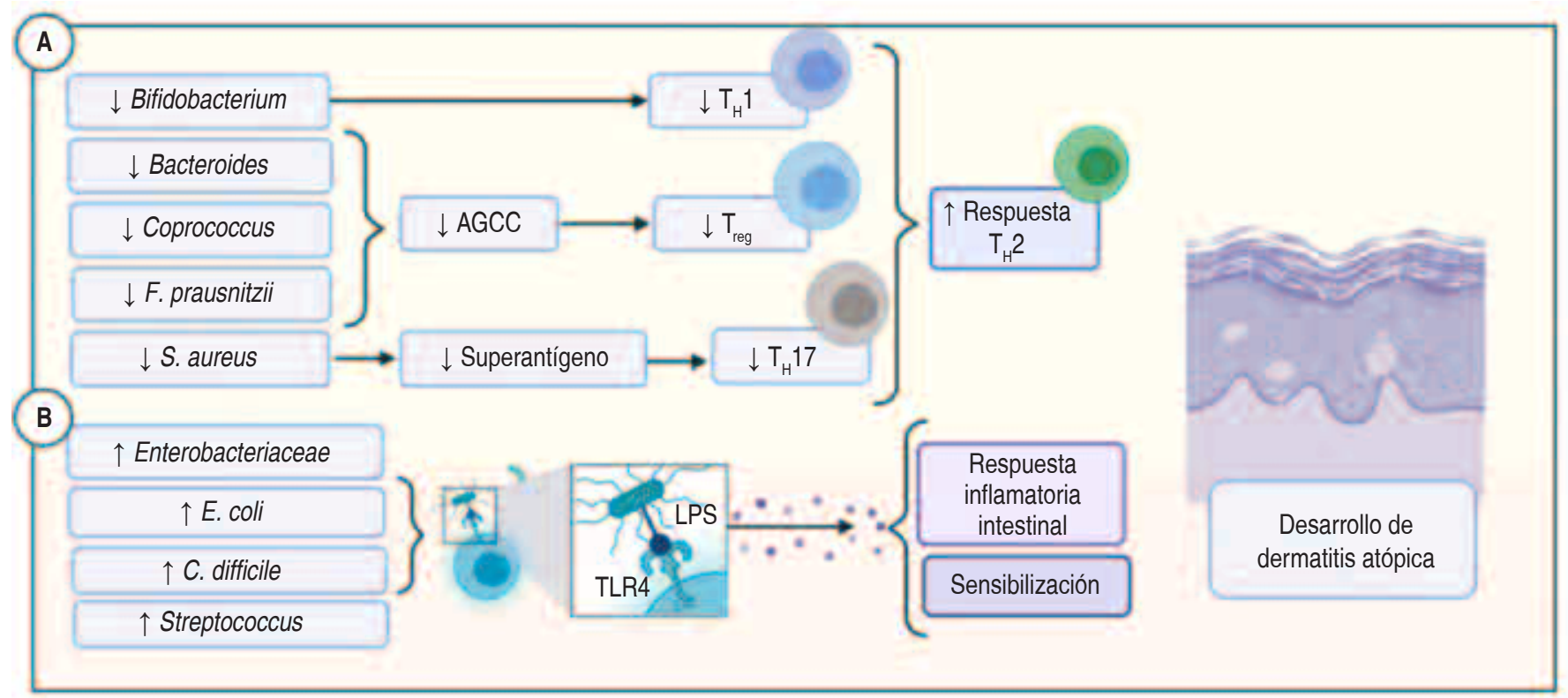

Figura 1: Microorganismos implicados en el desarrollo de dermatitis atópica. A) En la dermatitis atópica se observa disminución de Bifidobacterium, que estimula la producción de $T_{H}$; la disminución de bacterias productoras de ácidos grasos de cadena corta no ayudan a una adecuada producción de células $T_{\text {reg; }}$ S. aureus puede producir una respuesta tipo $T_{H}$ 17. B) Las colonias de Enterobacteriaceae (E. coli), C. difficile y Streptococus se ven aumentadas en la dermatitis atópica, E. coli puede evocar una respuesta inflamatoria intestinal gracias a los LPS.

AGCC = ácidos grasos de cadena corta, $T_{H} 1=$ célula $T$ cooperadora $1, T_{H} 2=$ célula $T$ cooperadora $2, T_{H} 17=$ célula $T$ cooperadora $17, T_{\text {reg: }}$ célula $T$ reguladora, $L P S=$ lipopolisacaridos, TLR4 = receptor 4 tipo toll.

antiinflamatorios; sin embargo, se ven alterados en los pacientes con DA. ${ }^{42}$

Las proteobacterias tienen lipopolisacáridos incorporados a la pared celular, esta endotoxina puede inducir una respuesta $T_{H} 1$ a través del sistema inmune innato, produciendo IL-2 a partir de monocitos y células dendríticas; la baja exposición a toxinas se ha asociado a mayor riesgo de DA. ${ }^{46}$ En recién nacidos, las proteobacterias se han relacionado con eccema asociado a $\operatorname{lgE} .{ }^{47}$

En una investigación, se encontró que la cantidad de Enterobacteriaceae se asocia negativamente con factor de necrosis tumoral alfa (TNF- $\alpha$ ) e IL-6 en niños con eczema asociado a $\operatorname{lgE}{ }^{45}$

Staphylococcus aureus (S. aureus) puede producir enterotoxinas activadoras de células T, llamados superantígenos, se asocia negativamente a la DA, por lo que podría tener efecto protector, activa la respuesta $T_{H} 17$, fortaleciendo la barrera intestinal. ${ }^{48}$ De manera contraria en la piel, el desequilibrio entre las respuestas $T_{H} 1 / T_{H} 2$, induce la producción de IL-4, IL-5 e IL-13, llevando a mayor producción de lgE y aumento en la unión de $S$. aureus a la piel con DA. ${ }^{49}$

La exposición temprana a microbios intestinales cambia el equilibrio $T_{H} 1 / T_{H} 2$ a un fenotipo $T_{H} 1$, la trans- formación de células $T$ vírgenes en diferentes tipos de células $T_{H}$, como $T_{H} 1, T_{H} 2$ y $T_{H} 17$ o células Forkhead box P3 (Foxp3) y $\mathrm{T}_{\text {reg }}$ depende en gran medida de la microbiota intestinal. Por el contrario, la ausencia de colonización bacteriana intestinal normal en las enfermedades atópicas, en especial durante el desarrollo del sistema inmunológico de la mucosa, empuja el equilibrio $T_{H} 1 / T_{H} 2$ hacia una respuesta $T_{H} 2$. Los mediadores derivados de células $T_{H} 2$, como IL-4, IL-5 e IL-13 inducen el cambio de inmunoglobulina a $\operatorname{lgE}$, manteniendo así la respuesta alérgica. ${ }^{26,50,51}$

\section{Disrupción de la barrera intestinal}

La disrupción de la barrera intestinal en la DA permite a las células dendríticas entrar en el lumen y capturar antígenos. A diferencia de la alteración de la Claudina-1 en la piel, la permeabilidad intestinal se ve influenciada por alteración en la Claudina-2 en pacientes atópicos, debido a la desregulación de la vía de la IL-4/IL-13 que aumenta la expresión de esta molécula. ${ }^{15,52,53} \mathrm{En}$ las células $T$ periféricas se presenta aumento en la proporción de células CD4+ que producen IL-4 y hay reducción en la cantidad de células Foxp3 + CD25, 
esto sugiere que la disbiosis del microbioma intestinal neonatal, asociada a la atopia, impulsa la disfunción de las células T CD4+. ${ }^{54}$

La permeabilidad intestinal alterada en la DA promueve la inflamación de la piel al permitir la penetración de toxinas y microbios en la circulación sistémica y la sensibilización por IgE; el aumento en la penetración de los antígenos podría resultar en la fagocitosis de los antígenos alimentarios por los macrófagos y su exposición a las células T en los ganglios linfáticos, activando la respuesta sistémica $T_{H} 2$; a medida que llegan a la piel, se inicia una fuerte respuesta $T_{H} 2$, lo que provoca daño tisular significativo, los niveles de células $\mathrm{T}_{\text {reg }}$ están disminuidas o son ineficientes para modular la respuesta inflamatoria. ${ }^{2,33,44,49}$

Estos mecanismos pueden estar sustentados por la activación alterada de los genes de microbios involucrados en la regulación inmune, por la posible pérdida de bacterias tolerogénicas, y equilibrio alterado de los metabolitos (AGCC) de las bacterias con la consiguiente reducción de las células $T_{\text {reg, }}$ lo anterior en el intestino puede inducir a una expansión de CD4+expresando receptores de célula T contra las bacterias de la microbiota resultando en inflamación ${ }^{2,55,56}$ (Figura 2).

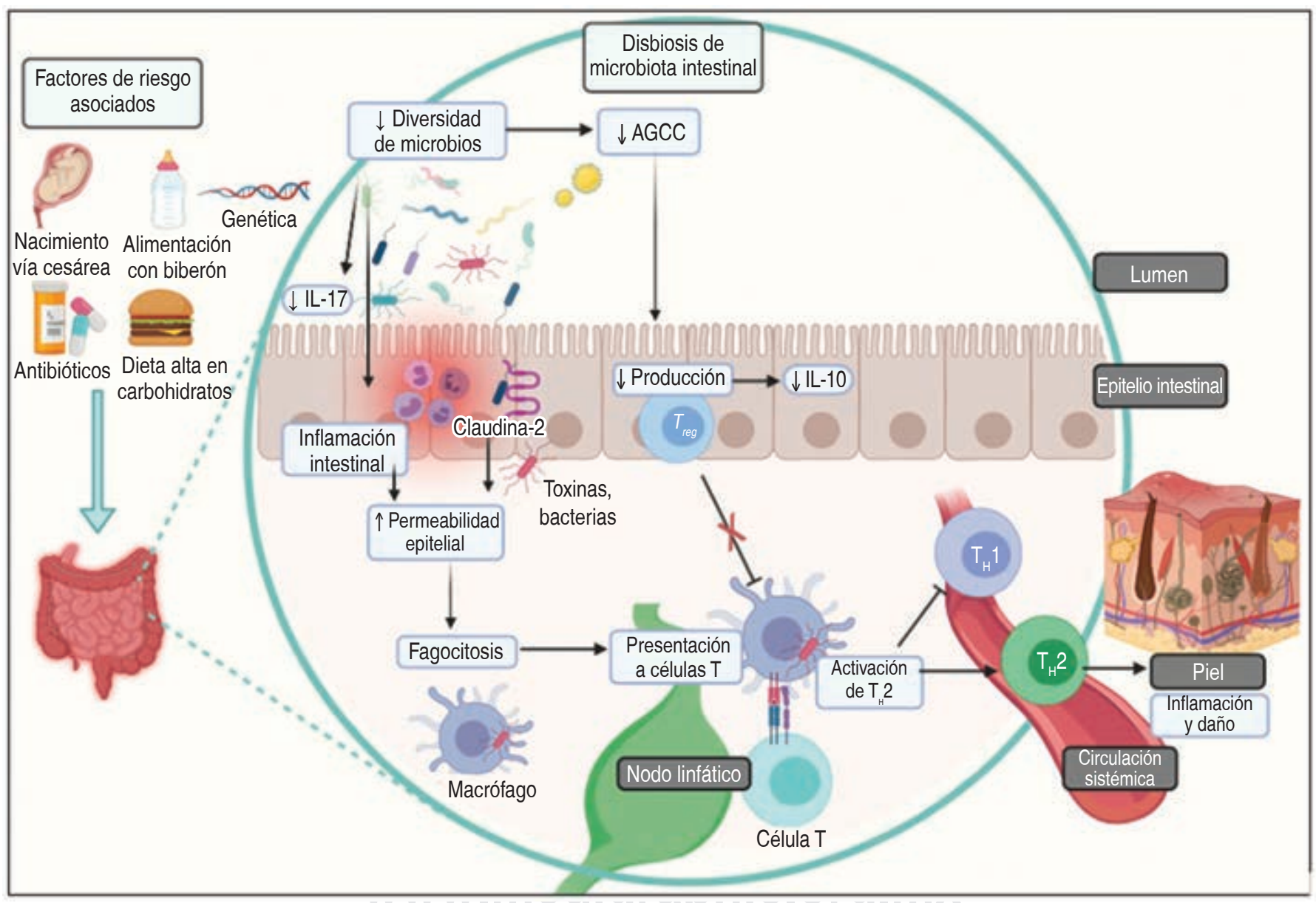

Figura 2: Patogénesis de la disbiosis intestinal en la dermatitis atópica. Factores asociados a disbiosis intestinal: nacimiento vía cesárea, alimentación con biberón, genética, uso de antibióticos, dieta alta en carbohidratos. La disminución en la diversidad de microbios en el lumen intestinal condicionan la disminución de microorganismos productores de ácidos grasos de cadena corta, esto provoca disminución de las células $T_{\text {reg }}$ así como disminución en su respuesta antiinflamatoria (IL-10). Por otro lado, la inflamación intestinal inducida por la disbiosis crea aumento en la permeabilidad intestinal, así como la posible alteración en la claudina-2, facilitando el paso de toxinas y bacterias a través del epitelio intestinal, son fagocitados por macrófagos y llevados al nodo linfático, donde se lleva a cabo la presentación a las células $T$, activándose una repuesta $T_{H} 2$, que pasa a la circulación sistémica llegando a la piel causando inflamación y daño.

AGCC = ácidos grasos de cadena corta, $T_{H} 1=$ célula $T$ cooperadora 1, $T_{H} 2=$ célula $T$ cooperadora 2, $T_{\text {reg }}=$ célula $T$ reguladora, LPS = lipopolisacáridos, IL-10: interleucina-10. 


\section{IMPACTO DE LOS PROBIÓTICOS}

Los probióticos son bacterias vivas y levaduras. Se ha encontrado que los probióticos modulan la respuesta inmune, mejoran la función de la barrera intestinal y contribuyen a la prevención y tratamiento de enfermedades atópicas como la DA. ${ }^{57,58}$ Bifidobacterium grampositivos y Lactobacillus son familias de probióticos que disminuyen la inflamación y producen AGCC. Los probióticos en la DA modulan el sistema inmunológico, fomentando la inducción y diferenciación de $T_{\text {reg }}$, células dendríticas reguladoras $(\mathrm{rDC})$ que expresan citocinas antiinflamatorias (TGF- $\beta$ e IL-10), el tipo de señalización que se produce depende de la cepa probiótica. ${ }^{59,60}$

Rosenfeldt y colaboradores demostraron en un estudio doble ciego la disminución en la excreción de lactulosa tras la administración de probióticos de lactobacilos, sugiriendo un mejoramiento en la barrera intestinal. ${ }^{58}$ Un metaanálisis reciente concluyó que la suplementación con probióticos durante la gestación y la primera infancia parece ser beneficiosa contra el desarrollo de la DA en los niños. ${ }^{60}$

Una revisión sistemática de 44 estudios expuso que, en la mitad de ellos, el uso de probióticos tuvo efecto positivo en la severidad de la DA, a pesar de presentar alteraciones en la composición de la microbiota intestinal; en los demás no se observó efecto benéfico de los probióticos. En la actualidad, su uso es controvertido para el tratamiento de la DA, ya que no hay evidencias claras de beneficio real y constante, además, no ha sido estudiado de manera adecuada el uso de un perfil seguro en el tratamiento, así como las dosis utilizadas y el tiempo de administración, y si existen efectos a largo plazo, ya que la mayoría de investigaciones realizadas evalúan el resultado en un tiempo corto. ${ }^{60}$

\section{CONCLUSIONES}

Las superficies mucosas con deterioro de la función y alteración de la microbiota, como en el intestino, podrían representar un sitio desencadenante de autoinmunidad por la generación de neoantígenos en condiciones disbióticas; aún está en debate si una barrera intestinal alterada facilita un mayor espectro de trastornos de hipersensibilización, como en la DA..$^{15,56}$

Los recientes avances tecnológicos en la secuenciación de próxima generación han fortalecido la capacidad para describir la composición del microbioma intestinal humano. La comprensión de la microbiota intestinal en las dermatosis inmunomediadas podría sugerir trata- mientos adicionales además de las terapias convencionales. Los estudios de hipersensibilización, como en la dermatitis atópica, sobre el efecto de una microbiota alterado (e.g. a través del trasplante fecal) han mostrado resultados prometedores en la aterosclerosis y algunos tipos de cáncer. ${ }^{2,33}$ Entre las estrategias que podrían producir mayor efecto protector, la lactancia materna parece tener influencia a largo plazo.

\section{COMENTARIO}

Si bien la microbiota intestinal infantil parece influir en el desarrollo inmunológico y en las vías metabólicas que conllevan al desarrollo de la dermatitis atópica, se requieren más estudios sobre el momento adecuado de las intervenciones y las complejas interacciones entre el sistema inmunológico infantil y la microbiota intestinal para traducir estos hallazgos en estrategias preventivas necesarias para reducir el riesgo de DA. Aún existe controversia en cuanto al rol de la disbiosis intestinal en el desarrollo de DA; sin embargo, publicaciones actuales demuestran un posible camino para entender mejor la patogenia de la enfermedad.

\section{REFERENCIAS}

1. Lloyd-Price J, Abu-Ali G, Huttenhower C. The healthy human microbiome. Genome Med. 2016; 8: 51.

2. Colucci R, Moretti S. Implication of human bacterial gut microbiota on immune-mediated and autoimmune dermatological diseases and their comorbidities: a narrative review. Dermatol Ther (Heidelb). 2021; 11: 363-384.

3. Zhu TH, Zhu TR, Tran KA, Sivamani RK, Shi VY. Epithelial barrier dysfunctions in atopic dermatitis: a skin-gut-lung model linking microbiome alteration and immune dysregulation. $\mathrm{Br} J$ Dermatol. 2018; 179: 570-581.

4. Thursby $\mathrm{E}$, Juge N. Introduction to the human gut microbiota. Biochem J. 2017; 474: 1823-1836.

5. Selber-Hnatiw S, Rukundo B, Ahmadi M, Akoubi H, Al-Bizri H, Aliu $A F$ et al. Human gut microbiota: toward an ecology of disease. Front Microbiol. 2017; 8: 1265.

6. Lee HJ, Lee SH. Epidermal permeability barrier defects and barrier repair therapy in atopic dermatitis. Allergy Asthma Immunol Res. 2014; 6: 276-287.

7. Rea D, Coppola G, Palma G, Barbieri A, Luciano A, Del Prete P et al. Microbiota effects on cancer: from risks to therapies. Oncotarget 2018; 9: 17915-17927.

8. Opazo MC, Ortega-Rocha EM, Coronado-Arrázola I, Bonifaz LC, Boudin H, Neunlist M, Bueno SM et al. Intestinal microbiota influences non-intestinal related autoimmune diseases. Front Microbiol. 2018; 9 : 432.

9. Lee SY, Lee E, Park YM, Hong SJ. Microbiome in the gut-skin axis in atopic dermatitis. Allergy Asthma Immunol Res. 2018; 10: 354-362. 
10. Flohr C, Mann J. New insights into the epidemiology of childhood atopic dermatitis. Allergy. 2014; 69: 3-16.

11. Kapur S, Watson W, Carr S. Atopic dermatitis. Allergy Asthma Clin Immunol. 2018; 14: 52.

12. Grice EA, Segre JA. The human microbiome: our second genome. Annu Rev Genomics Hum Genet. 2012; 13: 151-170.

13. Natividad JM, Verdu EF. Modulation of intestinal barrier by intestinal microbiota: Pathological and therapeutic implications. Pharmacol Res. 2013; 69: 42-51.

14. LeBlanc JG, Milani C, de Giori GS, Sesma F, van Sinderen D, Ventura M. Bacteria as vitamin suppliers to their host: a gut microbiota perspective. Curr Opin Biotechnol. 2013; 24: 160-168.

15. Belkaid $Y$, Harrison OJ. Homeostatic immunity and the microbiota. Immunity. 2017; 46: 562-576.

16. Martin R, Makino H, Cetinyurek YA, Ben-Amor K, Roelofs M, Ishikawa $E$ et al. Early-life events, including mode of delivery and type of feeding, siblings and gender, shape the developing gut microbiota. PLoS One. 2016; 11: e0158498.

17. Gensollen T, lyer SS, Kasper DL, Blumberg RS. How colonization by microbiota in early life shapes the immune system. Science. 2016; 352: 539-544.

18. Rodríguez JM, Murphy K, Stanton C, Ross RP, Kober OI, Juge N et al. The composition of the gut microbiota throughout life, with an emphasis on early life. Microb Ecol Health Dis. 2015; 26: 26050.

19. Arrieta MC, Stiemsma LT, Amenyogbe N, Brown EM, Finlay B. The intestinal microbiome in early life: health and disease. Front Immunol. 2014; 5: 427.

20. Martin R, Makino H, Cetinyurek YA, Ben-Amor K, Roelofs M, Ishikawa $E$ et al. Early-life events, including mode of delivery and type of feeding, siblings and gender, shape the developing gut microbiota. PLoS One. 2016; 11: e0158498.

21. Backhed F, Roswall J, Peng Y, Feng Q, Jia H, Kovatcheva-Datchary $P$ et al. Dynamics and stabilization of the human gut microbiome during the first year of life. Cell Host Microbe. 2015; 17: 690-703.

22. Hassoun $Y$, James $C$, Bernstein DI. The effects of air pollution on the development of atopic disease. Clin Rev Allergy Immunol. 2019; 57 : 403-414.

23. Gomez de Agüero M, Ganal-Vonarburg SC, Fuhrer T, Rupp S, Uchimura Y, Li H, Steinert A et al. The maternal microbiota drives early postnatal innate immune development. Science. 2016; 351: 1296-1302.

24. Gill SR, Pop M, Deboy RT, et al. Metagenomic analysis of the human distal gut microbiome. Science. 2006; 312: 1355-1359.

25. Russell AB, Wexler AG, Harding BN, Whitney JC, Bohn AJ, Goo YA et al. A type VI secretion-related pathway in Bacteroidetes mediates interbacterial antagonism. Cell Host Microbe. 2014; 16: 227-236.

26. Fujimura KE, Sitarik AR, Havstad S, Lin DL, Levan S, Fadrosh D et al. Neonatal gut microbiota associates with childhood multisensitized atopy and T cell differentiation. Nat Med. 2016; 22: 1187-1191.

27. Qin J, Li R, Raes J, Arumugam M, Burgdorf KS, Manichanh C et al. A human gut microbial gene catalogue established by metagenomic sequencing. Nature. 2010; 464: 59-65.

28. Gronlund MM, Gueimonde M, Laitinen K, Kociubinski G, Gronroos T, Salminen $S$ et al. Maternal breast-milk and intestinal bifidobacteria guide the compositional development of the Bifidobacterium microbiota in infants at risk of allergic disease. Clin Exp Allergy. 2007; 37: 1764-1772.

29. Bezirtzoglou E, Tsiotsias A, Welling GW. Microbiota profile in feces of breast and formula-fed newborns by using fluorescence in situ hybridization (FISH). Anaerobe. 2011; 17: 478-482.

30. Ardeshir A, Narayan NR, Méndez-Lagares G, Lu D, Rauch M, Huang $Y$ et al. Breast-fed and bottle-fed infant rhesus macaques develop distinct gut microbiotas and immune systems. Sci Transl Med. 2014; 6: 252ra120.

31. Narayan NR, Méndez-Lagares G, Ardeshir A, Lu D, Van Rompay KK, Hartigan-O'Connor DJ. Persistent effects of early infant diet and associated microbiota on the juvenile immune system. Gut Microbes. 2015; 6: 284-289.

32. Kim JE, Kim HS. Microbiome of the skin and gut in atopic dermatitis (AD): understanding the pathophysiology and finding novel management strategies. J Clin Med. 2019; 8: 444.

33. Biedermann T, Skabytska Y, Kaesler S, Volz T. Regulation of T cell immunity in atopic dermatitis by microbes: the yin and yang of cutaneous inflammation. Front Immunol. 2015; 6: 353.

34. Wang M, Karlsson C, Olsson C, Adlerberth I, Wold AE, Strachan DP et al. Reduced diversity in the early fecal microbiota of infants with atopic eczema. J Allergy Clin Immunol. 2008; 121: 129-134.

35. Abrahamsson TR, Jakobsson HE, Andersson AF, Bjorkstén B, Engstrand $L$, Jenmalm MC. Low diversity of the gut microbiota in infants with atopic eczema. J Allergy Clin Immunol. 2012; 129: 434-40, 440.e1-2.

36. Park YM, Lee SY, Kang MJ, Kim BS, Lee MJ, Jung SS et al. Imbalance of Gut Streptococcus, Clostridium, and Akkermansia determines the natural course of atopic dermatitis in infant. Allergy Asthma Immunol Res. 2020; 12: 322-337.

37. Nylund L, Nermes M, Isolauri E, Salminen S, de Vos WM, Satokari R. Severity of atopic disease inversely correlates with intestinal microbiota diversity and butyrate-producing bacteria. Allergy. 2015; 70: 241-244.

38. Reddel S, Del Chierico F, Quagliariello A, Giancristoforo S, Vernocchi $\mathrm{P}$, Russo A et al. Gut microbiota profile in children affected by atopic dermatitis and evaluation of intestinal persistence of a probiotic mixture. Sci Rep. 2019; 9: 4996.

39. Watanabe S, Narisawa Y, Arase S, Okamatsu H, Ikenaga T, Tajiri Y, Kumemura M. Differences in fecal microflora between patients with atopic dermatitis and healthy control subjects. J Allergy Clin Immunol. 2003; 111: 587-591.

40. Song H, Yoo Y, Hwang J, Na YC, Kim HS. Faecalibacterium prausnitzii subspecies-level dysbiosis in the human gut microbiome underlying atopic dermatitis. J Allergy Clin Immunol. 2016; 137: 852-860.

41. Rossi O, van Berkel LA, Chain F, Tanweer KM, Taverne N, Sokol H, Duncan $\mathrm{SH}$ et al. Faecalibacterium prausnitzii A2-165 has a high capacity to induce IL-10 in human and murine dendritic cells and modulates T cell responses. Sci Rep. 2016; 6: 18507.

42. Penders J, Thijs C, van den Brandt PA, Kummeling I, Snijders B, Stelma F et al. Gut microbiota composition and development of atopic manifestations in infancy: the KOALA Birth Cohort Study. Gut. 2007; 56: 661-667.

43. Kang Y, Cai Y, Pan W. Change in gut microbiota for eczema: implications for novel therapeutic strategies. Allergol Immunopathol. 2018; 46: 281-290. 
44. Gehring U, Bolte G, Borte M, Bischof W, Fahlbusch B, Wichmann HE et al. Exposure to endotoxin decreases the risk of atopic eczema in infancy: a cohort study. J Allergy Clin Immunol. 2001; 108: 847-854.

45. West CE, Rydén P, Lundin D, Engstrand L, Tulic MK, Prescott SL. Gut microbiome and innate immune response patterns in IgE-associated eczema. Clin Exp Allergy. 2015; 45: 1419-1429.

46. Huang YJ, Marsland BJ, Bunyavanich S, O'Mahony L, Leung DY, Muraro A, Fleisher TA. The microbiome in allergic disease: current understanding and future opportunities-2017 PRACTALL document of the American Academy of Allergy, Asthma \& Immunology and the European Academy of Allergy and Clinical Immunology. J Allergy Clin Immunol. 2017; 139: 1099-1110.

47. Belkaid $Y$, Hand TW. Role of the microbiota in immunity and inflammation. Cell. 2014; 157: 121-141.

48. Cosmi L, Liotta F, Maggi E, Romagnani S, Annunziato F. Th17 and non-classic Th1 cells in chronic inflammatory disorders: two sides of the same coin. Int Arch Allergy Immunol. 2014; 164: 171-177.

49. Vernocchi P, Del Chierico F, Fiocchi AG, El Hachem M, Dallapiccola $\mathrm{B}$, Rossi $\mathrm{P}$ et al. Understanding probiotics' role in allergic children: the clue of gut microbiota profiling. Curr Opin Allergy Clin Immunol. 2015; 15: 495-503.

50. Kubo A, Nagao K, Amagai M. Epidermal barrier dysfunction and cutaneous sensitization in atopic diseases. J Clin Invest. 2012; 122: 440-447.

51. Georas S, Rezaee F. Epithelial barrier function: at the front line of asthma immunology and allergic airway inflammation. J Allergy Clin Immun. 2014; 134: 509-520.

52. Kamada N, Seo SU, Chen GY, Núñez G. Role of the gut microbiota in immunity and inflammatory disease. Nat Rev Immunol. 2013; 13: 321-335.

53. De Oliveira GLV, Leite AZ, Higuchi BS, Gonzaga MI, Mariano VS. Intestinal dysbiosis and probiotic applications in autoimmune diseases. Immunology. 2017; 152: 1-12.

54. Lin RJ, Qiu LH, Guan RZ, Hu SJ, Liu YY, Wang GJ. Protective effect of probiotics in the treatment of infantile eczema. Exp Ther Med. 2015; 9: 1593-1596.
55. Zhao M, Shen $\mathrm{C}, \mathrm{Ma} \mathrm{L}$. Treatment efficacy of probiotics on atopic dermatitis, zooming in on infants: a systematic review and metaanalysis. Int J Dermatol. 2018; 57: 635-641.

56. Kwon HK, Lee CG, So JS, Chae CS, Hwang JS, Sahoo A et al. Generation of regulatory dendritic cells and CD4+Foxp3+ T cells by probiotics administration suppresses immune disorders. Proc Natl Acad Sci USA. 2010; 107: 2159-2164.

57. Karimi K, Inman MD, Bienenstock J, Forsythe P. Lactobacillus reuteriinduced regulatory $T$ cells protect against an allergic airway response in mice. Am J Respir Crit Care Med. 2009; 179: 186-193.

58. Rosenfeldt V, Benfeldt E, Valerius NH, Paerregaard A, Michaelsen KF. Effect of probiotics on gastrointestinal symptoms and small intestinal permeability in children with atopic dermatitis. J Pediatr. 2004; 145: 612-616.

59. Pothmann A, Illing T, Wiegand C, Hartmann AA, Elsner P. The microbiome and atopic dermatitis: a review. Am J Clin Dermatol. 2019; 20: 749-761.

60. Petersen EBM, Skov L, Thyssen JP, Jensen P. Role of the gut microbiota in atopic dermatitis: a systematic review. Acta Derm Venereol. 2019; 99: 5-11.

\section{Conflicto de intereses: Ninguno.}

\author{
Correspondencia: \\ Martha Alejandra Morales-Sánchez, MSc. \\ Centro Dermatológico «Dr. Ladislao de la Pascua». \\ Dr. Vértiz Núm. 464, \\ Col. Buenos Aires, 06780, \\ Alcaldía Cuauhtémoc, Ciudad de México, México. \\ Tel: 5555387033 \\ E-mail: marthamoralessanchez@gmail.com
}

